\title{
Left ventricular dyssynchrony assessment by phase analysis from gated PET-FDG scans
}

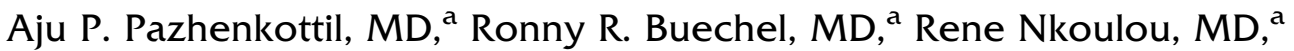 \\ Jelena-Rima Ghadri, MD, a Bernhard A. Herzog, MD, a Lars Husmann, MD, ${ }^{a}$ \\ Mathias Wolfrum, $M D,{ }^{\text {a }}$ Silke $M$. Küest, $M D,{ }^{a}$ Michael Fiechter, $M D$, ${ }^{a, b}$ \\ Oliver Gaemperli, MD, a and Philipp A. Kaufmann, $M D^{a, b}$
}

Background. The outcome of patients with severe ischaemic left ventricular (LV) dysfunction is determined by the extent of myocardial viability and the presence of $\mathrm{LV}$ dyssynchrony. We aimed at assessing both parameters from the same imaging method, i.e. gated positron emission tomography (PET) F18-fluorodeoxyglucose (FDG) scans.

Methods. Phase analysis from Emory Cardiac Toolbox was applied on gated PET-FDG scans to assess histogram bandwidth and standard deviation (SD) as a measure of LV dyssynchrony in 30 heart failure patients (mean ejection fraction: $30.2 \% \pm 13.8 \%$ ) referred for the evaluation of myocardial viability. Cut-off values from single-photon emission computed tomography myocardial perfusion imaging (SPECT-MPI) best predicting cardiac resynchronization therapy (CRT) response served as standard of reference (bandwidth $<135^{\circ}$; phase SD $<4^{\circ}$ ). Severe $L V$ dyssynchrony was diagnosed if both SPECT-MPI values were above these limits. Intraclass correlation and clinical agreement in detection of severe LV dyssynchrony by PET vs SPECT were assessed.

Results. There was a significant correlation between PET-FDG and SPECT-MPI for bandwidth $(r=0.88, P<.001)$ and phase SD $(r=0.88, P<.001)$ resulting in an excellent clinical agreement between the two methods of $93 \%$.

Conclusions. Accurate LV dyssynchrony assessment by phase analysis of gated PET-FDG scans is feasible, allowing assessing myocardial viability and severe $\mathrm{LV}$ dyssynchrony in one scan. (J Nucl Cardiol 2011;18:920-5.)

Key Words: Left ventricular dyssynchrony $\cdot$ phase analysis $\cdot$ myocardial perfusion imaging $\cdot$ myocardial viability

\section{INTRODUCTION}

Coronary artery disease (CAD) is one of the leading causes of morbidity and mortality in western countries. Patients with extensive coronary artery disease (CAD) are at higher risk to develop heart failure and left ventricular (LV) dysfunction.

From the Cardiac Imaging, ${ }^{\text {a }}$ University Hospital Zurich, Zurich, Switzerland; and Zurich Center for Integrative Human Physiology, University of Zurich, Zurich, Switzerland.

Aju P. Pazhenkottil and Ronny R. Buechel shared first authorship.

Received for publication Apr 26, 2010; final revision accepted May 27, 2011.

Reprint requests: Philipp A. Kaufmann, MD, Cardiac Imaging, University Hospital Zurich, Ramistrasse 100, 8091 Zurich, Switzerland; pak@usz.ch.

$1071-3581 / \$ 34.00$

Copyright (C) 2011 American Society of Nuclear Cardiology.

doi:10.1007/s12350-011-9411-y
In patients with severe ischaemic LV dysfunction revascularization of viable segments guided by myocardial PET-FDG findings has been shown to improve outcome. ${ }^{1-4}$ Recently, it has been shown that not only myocardial viability but also severe $\mathrm{LV}$ dyssynchrony is an independent predictor of perioperative outcome. ${ }^{5,6}$ Therefore, it has been advocated that the assessment of myocardial viability and LV dyssynchrony should be a routine part of the preoperative evaluation of these patients. $^{5}$

Moreover, studies showed that the presence of LV dyssynchrony as well as the extent of scar tissue and viable myocardium is directly related to the response to cardiac resynchronization therapy (CRT). Therefore, it has been stated that the evaluation for viability and LV dyssynchrony should be considered in the selection process for CRT. ${ }^{7-10}$

For the accurate assessment of LV dyssynchrony, different imaging methods, such as tissue Doppler imaging, multigated blood pool ventriculography 
acquisition (MUGA) and magnetic resonance imaging (MRI) have been developed. For long time, MUGA has been seen as the most accurate and reproducible noninvasive technique. ${ }^{11}$ Lately, phase analysis based on Fourier harmonic function on gated single-photonemission computed tomography myocardial perfusion imaging (SPECT-MPI) has been suggested as a suitable option for LV dyssynchrony assessment. ${ }^{12,13}$

As PET-FDG is considered the most specific tool for evaluation of viable tissue in ischaemic LV dysfunction where LV dyssynchrony has emerged as independent predictor of outcome, the assessment of the latter by gated PET-FDG appears pertinent. Thus, the aim of this study was to validate the assessment of LV dyssynchrony from phase analysis from gated PET-FDG scans vs previously established phase analysis from gated SPECT-MPI as standard of reference. ${ }^{12,13}$

\section{MATERIALS AND METHODS}

This study includes 30 consecutive patients with known CAD who were referred for evaluation of viable myocardium by SPECT-MPI/PET-FDG because of severe ischaemic LV dysfunction. Patients were included if they had signed informed consent authorizing their records to be included in our research registry.

\section{SPECT-MPI Image Acquisition and Interpretation}

The SPECT-MPI acquisition was performed approximately 1 hour after injection of $900 \mathrm{MBq}{ }^{99 \mathrm{~m}} \mathrm{Tc}$ tetrofosmin at rest on a Ventri dual-head camera (Ventri, GE Healthcare) with a low-energy, high-resolution collimator, a $20 \%$ symmetric window at $140 \mathrm{keV}$, a $64 \times 64$ matrix, and an elliptic orbit with step-and-shoot acquisition at $3^{\circ}$ intervals over $180^{\circ}$ arc $\left(45^{\circ}\right.$ right anterior oblique to $45^{\circ}$ left posterior oblique) with 30 steps (60 views). Scan time was set to 25 seconds per frame for stress and rest, resulting in a total acquisition time of 14 minutes 52 seconds (including inter-step rotation time) for each scan as recommended by the American Society of Nuclear Cardiology (ASNC). ${ }^{14}$ Images were reconstructed on a dedicated workstation using a standard iterative reconstruction algorithm with Ordered Subset Expectation Maximization (OSEM) with 2 iterations and 10 subsets. Standard short as well as vertical, and horizontal long axis, and polar maps of perfusion encompassing the entire left ventricle were analysed using QGS/QPS software. Effective radiation dose for the SPECT-MPI study was calculated as ${ }^{99 \mathrm{~m}} \mathrm{Tc}$ tetrofosmin activity times $7.9 \mathrm{mSv} / \mathrm{GBq}$.

\section{PET-FDG Image Acquisition and Interpretation}

Myocardial viability scans were acquired on a PET/ CT scanner using $250 \mathrm{MBq}$ of FDG. All patients were advised to fast for at least 6 hours before PET examination and received a standardized oral glucose load. ${ }^{15}$ After FDG injection, patients rested for 1 hour before image acquisition. Images were acquired on a Discovery (LS/RX) PET/CT scanner (GE Healthcare). CT attenuation maps were used for AC as previously reported. ${ }^{16}$ Acquired images were reconstructed using attenuation weighted-OSEM iterative reconstruction (2 iterations and 8 subsets). FDG uptake polar maps were equally derived using the Cedars QPS/QGS software package as for SPECT-MPI. Effective radiation dose for PET-FDG study was calculated as FDG activity times $0.019 \mathrm{mSv} /$ $\mathrm{MBq}$.

\section{Assessment of LV Dyssynchrony by SPECT- MPI and PET-FDG}

The Emory Cardiac Toolbox software (Emory University/Syntermed, Atlanta, GA) for phase analysis and assessment of LV dyssynchrony from SPECT-MPI has been extensively validated. ${ }^{12,13,17}$ The software was applied on both, gated PET-FDG and SPECT-MPI scans to assess phase histogram bandwidth and standard deviation (SD), which are the two parameters best allowing determination of presence vs absence of LV dyssynchrony. The previously established optimal cutoff value for the prediction of response to CRT, i.e. $135^{\circ}$ for histogram bandwidth and $43^{\circ}$ for phase $\mathrm{SD},{ }^{17}$ were used in this study. Severe LV dyssynchrony was considered to be present if both the parameters were above the cut-off value. All images were analysed by a reader who was blinded to the history of the patient.

\section{Statistical Analysis}

All statistical analyses were performed using SPSS software (SPSS 15.0, Chicago, IL, USA). Quantitative variables were expressed as mean \pm standard deviation, and categorical variables were expressed as frequencies and percentages. Means of histogram bandwidth and phase SD assessed by the two different imaging methods PET-FDG vs SPECT-MPI were compared using Wilcoxon signed-rank test. Intraclass correlation of the two parameters, histogram bandwidth and phase SD, from PET-FDG vs SPECT-MPI was performed. In addition, clinical agreement and Bland-Altman (BA) limits of agreement were calculated. A $P$ value of $<.05$ was considered to indicate statistical significance. 


\section{RESULTS}

In all 30 patients phase analysis of Emory Cardiac Toolbox was applied successfully using rest MPI scans and PET-FDG scans, respectively. Baseline characteristics of the entire study population are given in Table 1. The SPECT and FDG exams were performed in a time range between December 2007 and January 2010. The mean injected activity was $925 \pm 12 \mathrm{MBq}$ for the rest ${ }^{99 \mathrm{~m}} \mathrm{Tc}$-Tetrofosmin SPECT-MPI study and $252 \pm$ $20 \mathrm{MBq}$ for the PET-FDG study resulting in a total radiation dose of $7.3 \pm 1.0 \mathrm{mSv}$ for the SPECTMPI scan and $4.8 \pm 0.4 \mathrm{mSv}$ for the FDG exam, respectively.

SPECT-MPI revealed a perfusion defect in 27 patients. Of these, 19 patients had at least partially preserved FDG uptake, indicating dysfunctional but viable tissue.

Mean histogram bandwidth by SPECT-MPI and PET-FDG was $168.7^{\circ} \pm 78.4^{\circ}$ and $168.0^{\circ} \pm 66.7^{\circ}(P=$ n.s.), respectively. Mean phase SD by SPECT-MPI and PET-FDG was $52.7^{\circ} \pm 23.2^{\circ}$ and $57.3^{\circ} \pm 27.0^{\circ}$ $(P=$ n.s. $)$.

SPECT-MPI identified severe LV dyssynchrony in 18 patients with a mean histogram bandwidth of $225.2^{\circ} \pm 36.7^{\circ}$ and a mean phase SD of $68.7^{\circ} \pm 11.3^{\circ}$. Normal LV synchronicity or mild LV dyssynchrony was found in 12 patients with a mean bandwidth of $83.9^{\circ} \pm 32.8^{\circ}$ and a phase SD of $28.7^{\circ} \pm 13.1^{\circ}$. In 13 of these 18 patients there was a flow-metabolism mismatch, i.e. a perfusion defect in SPECT but preserved FDG uptake in PET in at least one segment. PET-FDG scans revealed severe LV dyssynchrony in 20 patients among which the 18 patients identified by SPECT-MPI. Mean histogram bandwidth was $204.9^{\circ} \pm 44.1^{\circ}$ and mean phase SD was $68.7^{\circ} \pm 17.0^{\circ}$. Mean values of normal or mild findings by PET-FDG were $94.2^{\circ} \pm 34.0^{\circ}$ and

Table 1. Baseline characteristics of study population $(n=30)$

\begin{tabular}{|c|c|}
\hline Male & $22(73.3 \%)$ \\
\hline \multicolumn{2}{|l|}{ Age (years) } \\
\hline Mean \pm SD (range) & $64 \pm 14(35-83)$ \\
\hline \multicolumn{2}{|c|}{ Body mass index $\left(\mathrm{kg} / \mathrm{m}^{2}\right)$} \\
\hline Mean \pm SD (range) & $25.8 \pm 3.7(20.7-34.9)$ \\
\hline \multicolumn{2}{|l|}{ Ejection fraction (\%) } \\
\hline Mean \pm SD, (range) & $30.2 \pm 13.8(11-55)$ \\
\hline Hypertension & $17(57 \%)$ \\
\hline Dyslipidemia & $10(33 \%)$ \\
\hline Diabetes & $8(27 \%)$ \\
\hline Smoking & $16(53 \%)$ \\
\hline Positive family history & $6(20 \%)$ \\
\hline
\end{tabular}
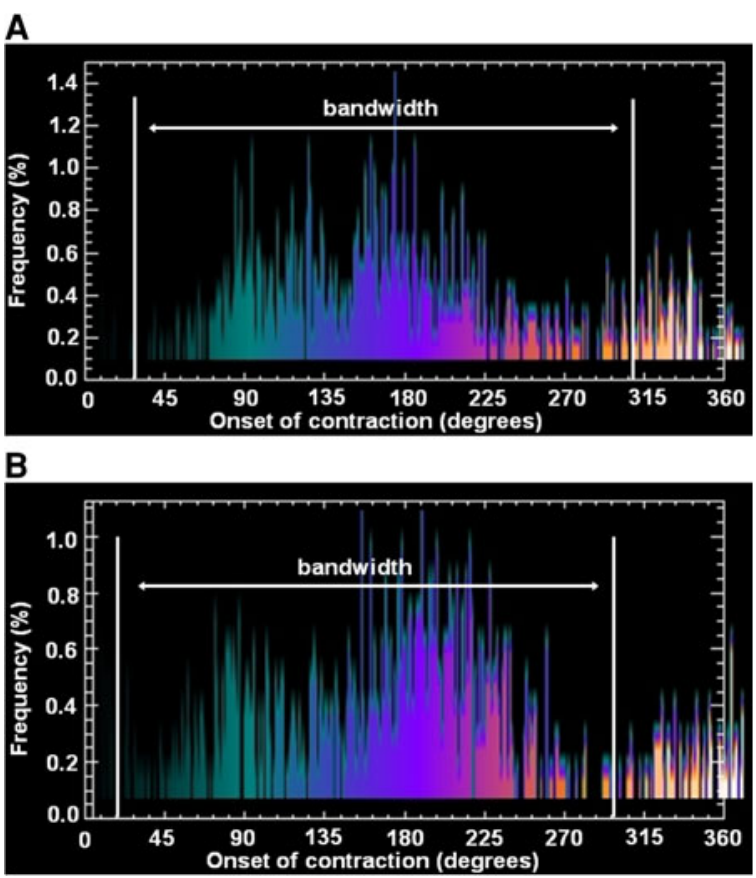

Figure 1. Phase analysis of gated SPECT-MPI (A) and PETFDG (B) in a patient with severe LV dyssynchrony reveals comparable results for histogram bandwidth $\left(281^{\circ}\right.$ and $\left.280^{\circ}\right)$ and phase $\mathrm{SD}\left(79^{\circ}\right.$ and $\left.81^{\circ}\right)$, respectively.

$34.5^{\circ} \pm 15.1^{\circ}$ for histogram bandwidth and SD, respectively. An example of dyssynchrony assessment from SPECT-MPI vs PET-FDG is illustrated in Figure 1.

There was a significant correlation between SPECTMPI data and PET-FDG scans for histogram bandwidth $(r=0.88, P<.001)$ and phase $\mathrm{SD}(r=0.88, P<$ $.001)$ (Figure 2). Sensitivity, specificity, PPV and NPV on a per-patient base were $100 \%, 83 \%, 90 \%$ and $100 \%$ for data sets from PET-FDG compared to SPECT-MPI as standard of reference. This resulted in an agreement of the two methods of $93 \%$.

\section{DISCUSSION}

This study investigates the use of PET-FDG scans to assess severe LV dyssynchrony in patients with ischaemic LV dysfunction referred for preoperative evaluation prior to CABG surgery. Our results reveal that LV dyssynchrony assessment from PET-FDG scans is feasible and accurate. This allows using the same scans performed for viability assessment also for assessment of LV dyssynchrony, which has recently shown to be an independent predictor of perioperative outcome in CABG surgery of patients with severe ischaemic $\mathrm{LV}$ dysfunction.

There are several therapeutic strategies, which have been considered for the treatment of patients with ischaemic heart failure. As the value of medical therapy 

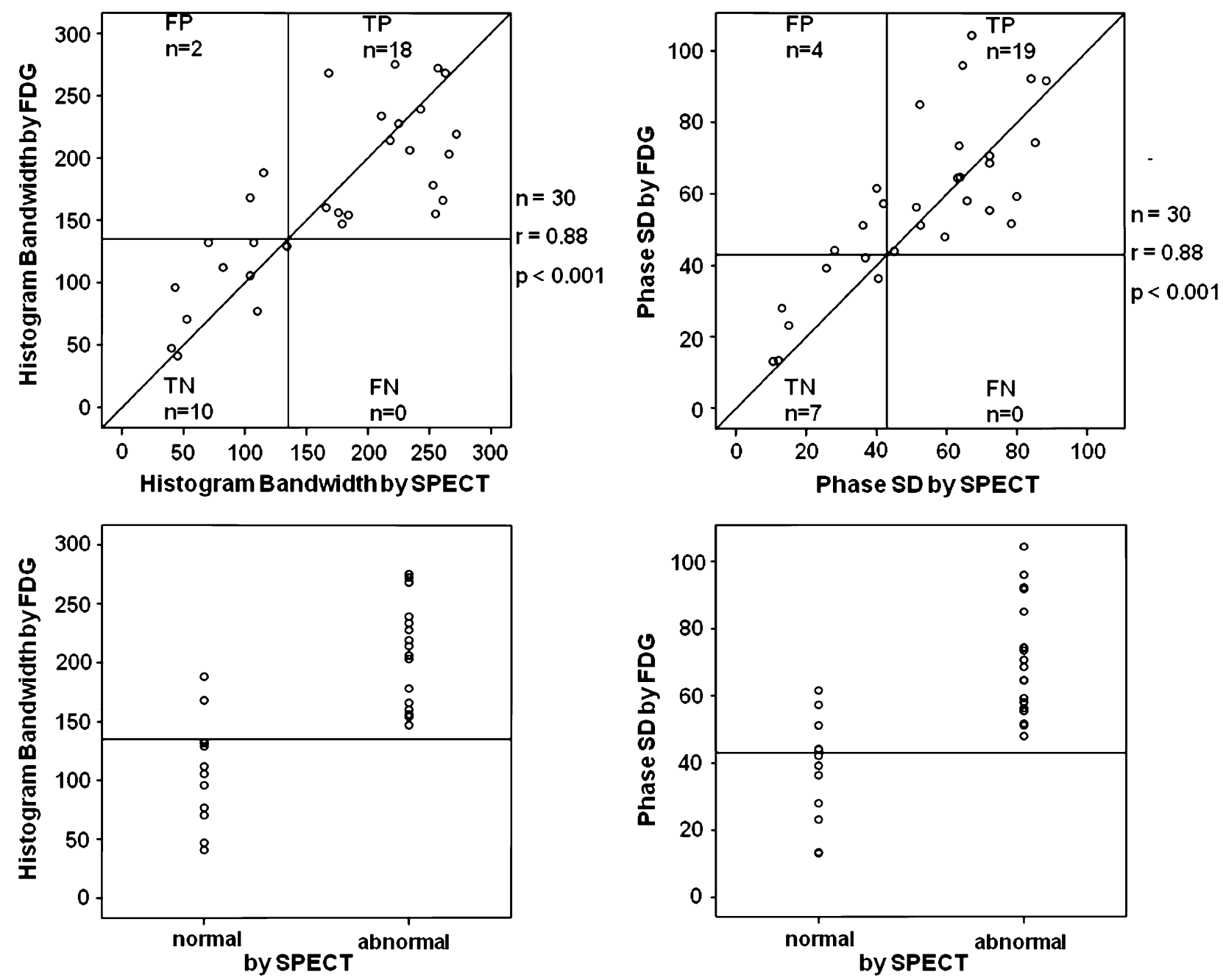

Figure 2. Correlation of histogram bandwidth (left) and phase SD (right) values between PETFDG vs SPECT-MPI (upper panel) and accuracy of PET-FDG to predict LV dyssynchrony (lower panel). Values greater than $43^{\circ}$ (for phase SD) and $135^{\circ}$ (for histogram bandwidth) from SPECTMPI were considered abnormal (according to Henneman et $\mathrm{al}^{17}$ ).

remains questionable, ${ }^{3,18}$ other modalities such as coronary revascularization and resynchronization have gained importance as valuable treatment option in these patients. CABG surgery improves survival in patients with chronic $\mathrm{CAD}$, and the presence of viability has been considered as one of the most important parameters of preoperative evaluation. Recently, a poor outcome in patients undergoing CABG surgery has been associated with the presence of severe LV dyssynchrony. ${ }^{5,6}$ Therefore, it has been suggested that both, viability as well as LV dyssynchrony should be routinely assessed before CABG surgery. ${ }^{5}$

CRT is an established therapy for patients with advanced heart failure. The technique improves heart failure symptoms, exercise capacity, and LV function, with a reduction in morbidity and mortality. ${ }^{19,20}$ However, $20 \%-30 \%$ of all patients receiving a CRT do not respond. The search of reasons for nonresponse to CRT has gained importance in recent investigations. The presence of LV dyssynchrony as well as the extent of scar tissue and viable myocardium has been identified as key parameters directly related to the response to $\mathrm{CRT}^{7-10}$ suggesting that the evaluation of patient selection for CRT should include these parameters.

PET-FDG is the only method for viability assessment which has been shown to improve the outcome of patients when it is used to assist management of severe LV dysfunction. ${ }^{1}$ A broad diversity of imaging techniques has been established for LV dyssynchrony assessment, such as echocardiography, especially with 
tissue Doppler imaging (TDI) and strain imaging, ${ }^{21,22}$ magnetic resonance imaging (MRI) and MUGA radionuclide ventriculography. TDI has emerged as one of the most widely used techniques but may be subject to interobserver variability in part related to observer experience. ${ }^{23}$ MUGA radionuclide ventriculography has overcome both of the above-mentioned possible disadvantages but has not been widely used lately. Recently, assessment of LV dyssynchrony by phase analysis of gated SPECT-MPI scans has been established. ${ }^{12}$ The evaluation with gated SPECT-MPI scans has potential benefits, such as the automated and reproducible assessment and moreover the possibility to gather information on myocardial perfusion at the same time. This study now shows that PET-FDG, which is used to assess viability, allows assessment of the two key parameters of severe LV dysfunction simultaneously. This enables comprehensive evaluation of patients before CABG surgery.

We acknowledge following limitations: first, this is a single-center study with only a limited population of 30 patients due to the pilot nature of this study. Second, the use of manual base and apex placement may lead to bias, especially in a population which typically has a high prevalence of perfusion defects. However, it has been described earlier that repeatability of phase analysis is higher when using manual placement compared to the automated technique ${ }^{24}$ justifying the manual placement tool for phase analysis. In addition, in patients with large fixed defects, the assessment of LV dyssynchrony by phase analysis from SPECT-MPI may be hampered, due to limitations in contour detection. By contrast, many of these segments had preserved FDG uptake potentially allowing better contour detection by PET-FDG scanning. This may have accounted for the fact that in the upper range of LV dyssynchrony there appears to be larger variability between the two methods (see Figure 2).

In conclusion, LV dyssynchrony assessment from PET-FDG scans is feasible and accurate, compared to the standard of reference SPECT-MPI, although our data may not support the interchangeable use of the two methods in patients with large perfusion defects.

\section{Acknowledgments}

The study was supported by a grant from the Swiss National Science Foundation and by the ZIHP (Zurich Center for Integrative Human Physiology, University of Zurich, Switzerland). We are grateful to Patrick von Schulthess, Ennio Mueller, Edlira Loga, Mirjam De Bloeme, Raji Kanagasabai, and Josephine Trinckauf for their excellent technical support.

\section{References}

1. Beanlands RS, Nichol G, Huszti E, Humen D, Racine N, Freeman $\mathrm{M}$, et al. F-18-fluorodeoxyglucose positron emission tomography imaging-assisted management of patients with severe left ventricular dysfunction and suspected coronary disease: A randomized, controlled trial (PARR-2). J Am Coll Cardiol 2007;50:2002-12.

2. Chareonthaitawee P, Gersh BJ, Araoz PA, Gibbons RJ. Revascularization in severe left ventricular dysfunction: The role of viability testing. J Am Coll Cardiol 2005;46:567-74.

3. Allman KC, Shaw LJ, Hachamovitch R, Udelson JE. Myocardial viability testing and impact of revascularization on prognosis in patients with coronary artery disease and left ventricular dysfunction: A meta-analysis. J Am Coll Cardiol 2002;39:1151-8.

4. Penicka M, Tousek P, De Bruyne B, Wijns W, Lang O, Madaric J, et al. Myocardial positive pre-ejection velocity accurately detects presence of viable myocardium, predicts recovery of left ventricular function and bears a prognostic value after surgical revascularization. Eur Heart J 2007;28:1366-73.

5. Penicka M, Bartunek J, Lang O, Medilek K, Tousek P, Vanderheyden M, et al. Severe left ventricular dyssynchrony is associated with poor prognosis in patients with moderate systolic heart failure undergoing coronary artery bypass grafting. J Am Coll Cardiol 2007;50:1315-23.

6. Maruskova M, Gregor P, Bartunek J, Tintera J. Penicka M Myocardial viability and cardiac dyssynchrony as strong predictors of perioperative mortality in high-risk patients with ischemic cardiomyopathy having coronary artery bypass surgery. J Thorac Cardiovasc Surg 2009;138:62-8.

7. Ypenburg C, Schalij MJ, Bleeker GB, Steendijk P, Boersma E, Dibbets-Schneider P, et al. Extent of viability to predict response to cardiac resynchronization therapy in ischemic heart failure patients. J Nucl Med 2006;47:1565-70.

8. Ypenburg C, Schalij MJ, Bleeker GB, Steendijk P, Boersma E, Dibbets-Schneider P, et al. Impact of viability and scar tissue on response to cardiac resynchronization therapy in ischaemic heart failure patients. Eur Heart J 2007;28:33-41.

9. Bax JJ, Bleeker GB, Marwick TH, Molhoek SG, Boersma E, Steendijk $\mathrm{P}$, et al. Left ventricular dyssynchrony predicts response and prognosis after cardiac resynchronization therapy. J Am Coll Cardiol 2004;44:1834-40.

10. Bax JJ, Marwick TH, Molhoek SG, Bleeker GB, van Erven L, Boersma E, et al. Left ventricular dyssynchrony predicts benefit of cardiac resynchronization therapy in patients with end-stage heart failure before pacemaker implantation. Am J Cardiol 2003;92: 1238-40.

11. Hesse B, Tagil K, Cuocolo A, Anagnostopoulos C, Bardies M, Bax $\mathrm{J}$, et al. EANM/ESC procedural guidelines for myocardial perfusion imaging in nuclear cardiology. Eur J Nucl Med Mol Imaging 2005;32:855-97.

12. Chen J, Garcia EV, Folks RD, Cooke CD, Faber TL, Tauxe EL, et al. Onset of left ventricular mechanical contraction as determined by phase analysis of ECG-gated myocardial perfusion SPECT imaging: Development of a diagnostic tool for assessment of cardiac mechanical dyssynchrony. J Nucl Cardiol 2005;12:687-95.

13. Henneman MM, Chen J, Ypenburg C, Dibbets P, Bleeker GB, Boersma E, et al. Phase analysis of gated myocardial perfusion single-photon emission computed tomography compared with tissue Doppler imaging for the assessment of left ventricular dyssynchrony. J Am Coll Cardiol 2007;49:1708-14.

14. Hansen CL, Goldstein RA, Akinboboye OO, Berman DS, Botvinick $\mathrm{EH}$, Churchwell $\mathrm{KB}$, et al. Myocardial perfusion and function: 
Single photon emission computed tomography. J Nucl Cardiol 2007; 14:e39-60.

15. Tillisch J, Brunken R, Marshall R, Schwaiger M, Mandelkern M, Phelps M, et al. Reversibility of cardiac wall-motion abnormalities predicted by positron tomography. N Engl J Med 1986;314:884-8.

16. Koepfli P, Hany TF, Wyss CA, Namdar M, Burger C, Konstantinidis AV, et al. CT attenuation correction for myocardial perfusion quantification using a PET/CT hybrid scanner. J Nucl Med 2004;45:537-42.

17. Henneman MM, Chen J, Dibbets-Schneider P, Stokkel MP, Bleeker GB, Ypenburg C, et al. Can LV dyssynchrony as assessed with phase analysis on gated myocardial perfusion SPECT predict response to CRT? J Nucl Med 2007;48:1104-11.

18. Bourque JM, Velazquez EJ, Borges-Neto S, Shaw LK, Whellan DJ. O'Connor CM Radionuclide viability testing: Should it affect treatment strategy in patients with cardiomyopathy and significant coronary artery disease? Am Heart J 2003;145:758-67.

19. Cleland JG, Daubert JC, Erdmann E, Freemantle N, Gras D, Kappenberger L, et al. The effect of cardiac resynchronization on morbidity and mortality in heart failure. $\mathrm{N}$ Engl $\mathrm{J} \mathrm{Med}$ 2005;352:1539-49.
20. Abraham WT, Fisher WG, Smith AL, Delurgio DB, Leon AR, Loh $\mathrm{E}$, et al. Cardiac resynchronization in chronic heart failure. N Engl J Med 2002;346:1845-53.

21. Bax JJ, Abraham T, Barold SS, Breithardt OA, Fung JW, Garrigue $\mathrm{S}$, et al. Cardiac resynchronization therapy: Part 1-issues before device implantation. J Am Coll Cardiol 2005;46:2153-67.

22. Suffoletto MS, Dohi K, Cannesson M, Saba S, Gorcsan J. 3rd Novel speckle-tracking radial strain from routine black-and-white echocardiographic images to quantify dyssynchrony and predict response to cardiac resynchronization therapy. Circulation 2006;113:960-8.

23. Chung ES, Leon AR, Tavazzi L, Sun JP, Nihoyannopoulos P, Merlino J, et al. Results of the Predictors of Response to CRT (PROSPECT) trial. Circulation 2008;117:2608-16.

24. Trimble MA, Velazquez EJ, Adams GL, Honeycutt EF, Pagnanelli RA, Barnhart HX, et al. Repeatability and reproducibility of phase analysis of gated single-photon emission computed tomography myocardial perfusion imaging used to quantify cardiac dyssynchrony. Nucl Med Commun 2008;29:374-81. 